\title{
A 2-Year, Longitudinal, Prospective Study of the Effects of Eltrombopag on Bone Marrow in Patients with Chronic Immune Thrombocytopenia
}

\author{
Russell K. Brynes ${ }^{a} \quad$ Raymond S.M. Wong ${ }^{\mathrm{e}} \quad$ Maung M. Thein $^{\mathrm{a}} \quad$ Kalpana K. Bakshib \\ Paul Burgess ${ }^{f}$ Dickens Theodore ${ }^{c}$ Attilio Orazi $^{d}$ \\ a Department of Pathology, Keck School of Medicine, University of Southern California, Los Angeles, CA, \\ bPCPS, GlaxoSmithKline, Collegeville, PA, COncology R\&D, GlaxoSmithKline, Research Triangle Park, NC, and \\ ${ }^{\mathrm{d}}$ Department of Pathology, Weill Cornell Medical College, New York, NY, USA; ${ }^{\mathrm{e}}$ Department of Medicine and \\ Therapeutics, Sir YK Pao Centre for Cancer, The Chinese University of Hong Kong, Shatin, Hong Kong, SAR China; \\ fResearch and Development, GlaxoSmithKline, Stockley Park, UK
}

\section{Keywords}

Bone marrow · Chronic immune thrombocytopenia ·

Collagen · Eltrombopag $\cdot$ Reticulin

\begin{abstract}
Background: The long-term effects of eltrombopag on bone marrow (BM) reticulin and/or collagen deposition in previously treated adults with chronic immune thrombocytopenia (ITP) were assessed. Methods: Three BM biopsies were collected at baseline and after 1 and 2 years of eltrombopag treatment. Specimens were centrally processed, stained for reticulin and collagen, independently reviewed by 2 hematopathologists, and rated according to the European Consensus 0-3 scale of marrow fibrosis (MF). Results: Of 162 patients enrolled, 93 completed all 3 protocol-specified BM biopsies. All patients with a baseline assessment were negative for collagen. Of 159 patients assessed at baseline, 150 (94\%) had normal reticulin (MF-0) and 9 (6\%) had minimally increased reticulin (MF-1). After 2 years, 83/93 patients (89\%) with BM biopsies had MF-0, 10 (11\%) had MF-1, and none had MF-2 or MF-3. Five out of 127 patients (4\%) at 1 year and 1 out of $93(1 \%)$ at 2 years had collagen deposition. None of the patients had clinical symptoms typical of BM dysfunction
\end{abstract}

\section{KARGER}

(c) 2016 S. Karger AG, Basel

E-Mail karger@karger.com

www.karger.com/aha or abnormalities of clinical concern based on white blood cell count or peripheral blood smear. Conclusion: For most patients with chronic ITP, eltrombopag is not associated with clinically relevant increases in BM reticulin or collagen formation.

(c) 2016 S. Karger AG, Basel

\section{Introduction}

The acquired disorder immune thrombocytopenia (ITP) results in low platelet counts due to an increase in platelet clearance [1], as well as an inhibition of megakaryocyte platelet release [2]. Mild reticulin has been shown to be present in the bone marrow (BM) of approximately $27-31 \%$ of hematologically normal patients $[3,4]$ and $28-52 \%$ of patients diagnosed with primary ITP $[5,6]$.

Eltrombopag, an oral nonpeptide thrombopoietin receptor agonist (TPO-RA), increases platelet counts in patients with chronic ITP [7-10]. However, TPO-RAs have

Clinicaltrials.gov identifier: NCT01098487. 
been associated with increases in reticulin fibers in the $\mathrm{BM}$ [11-13]. Whether the risk of BM fibrosis is due to the TPO-RAs themselves or to the underlying disease remains unclear. While BM biopsies can detect an increase in reticulin deposition in the BM, they are not routinely performed in the management of patients with chronic ITP. Although some studies have retrospectively reviewed biopsy specimens, prospectively collected data in patients with chronic ITP are limited. Additionally, inconsistencies in specimen preparation, staining, and pathologic analysis across institutions in previous studies have further confounded conclusions.

The purpose of this study was to prospectively investigate the potential effects of eltrombopag on the BM. Specifically, the study was designed to characterize any change in BM fibrosis in patients with previously treated persistent/chronic ITP from baseline to 1 and 2 years after initiating treatment with eltrombopag.

\section{Methods}

\section{Study Design}

This phase IV, open-label safety study was conducted at 28 centers in 11 countries from May 6, 2010, to May 6, 2014. The study was conducted according to the principles set forth in the 2008 Declaration of Helsinki. Written informed consent was obtained from each patient prior to the performance of any study-specific procedures. The study protocol was reviewed and approved by national, regional, or investigational center ethics committees or institutional review boards as appropriate. The primary objective was to assess the levels of BM reticulin and/or collagen fibers at baseline and to detect any changes after 1 and 2 years of treatment with eltrombopag in adult patients with persistent/chronic ITP. The secondary objective was the long-term safety and tolerability of oral eltrombopag treatment in patients with persistent/chronic ITP.

\section{Eligibility}

Adult patients with a diagnosis of persistent ITP (for $\geq 6$ months) or chronic ITP (for $\geq 12$ months) based on the American Society of Hematology/British Committee for Standards in Haematology guidelines [14-16], with no evidence of other disease that could cause thrombocytopenia, were eligible for study inclusion. Patients who had previously received eltrombopag or romiplostim must have completed treatment with these agents $\geq 6$ months before the screening BM biopsy. Patients treated with any other TPO-RA were not eligible. Patients were not eligible if their baseline BM biopsy had a marrow fibrosis (MF) reticulin grade of MF-3 (the highest grade according to the European Consensus scale) [17].

\section{Enrollment and Treatment}

The enrollment of patients previously exposed to a TPO-RA was limited to no more than $20 \%$ of the total study population. The duration of the screening period was up to 8 weeks. Eltrombopag
Table 1. AEs leading to discontinuation $(n=162)$

\begin{tabular}{ll}
\hline Event & $n(\%)$ \\
\hline Increased ALT level & $3(2)$ \\
Toxic hepatitis & $2(1)$ \\
Cerebral hemorrhage & $2(1)$ \\
Increased blood bilirubin & $2(1)$ \\
Hepatitis & $1(1)$ \\
Acute hepatitis & $1(1)$ \\
Hepatosplenomegaly & $1(1)$ \\
Cerebral venous thrombosis & $1(1)$ \\
Transient ischemic attack & $1(1)$ \\
Increased AST level & $1(1)$ \\
Acute respiratory distress syndrome & $1(1)$ \\
Cough & $1(1)$ \\
Pulmonary embolism & $1(1)$ \\
Gastrointestinal hemorrhage & $1(1)$ \\
Gingival bleeding & $1(1)$ \\
Nausea & $1(1)$ \\
Vomiting & $1(1)$ \\
Cardiovascular disorder & $1(1)$ \\
Vertigo & $1(1)$ \\
Chest pain & $1(1)$ \\
Myalgia & $1(1)$ \\
T cell lymphoma & $1(1)$ \\
Deep vein thrombosis & $1(1)$ \\
\hline
\end{tabular}

AEs, adverse events; ALT, alanine aminotransferase; AST, aspartate aminotransferase.

was administered for 2 years ( \pm 8 weeks) followed by a 4 -week follow-up period. The total study duration was up to 116 weeks. The starting dose of eltrombopag was $50 \mathrm{mg} /$ day, or $25 \mathrm{mg} /$ day for patients of East Asian ancestry. The dosing regimen of eltrombopag was then adjusted to a lower dose or a maximum dose of $75 \mathrm{mg}$ for each patient to maintain platelet counts within the clinically indicated range.

\section{Specimen Collection and Analysis}

Biopsy specimens were collected at baseline (before treatment) and after 1 and 2 years of treatment with eltrombopag, or at early withdrawal of treatment if the patient withdrew $>3$ months after the last BM biopsy. Fresh trephine biopsy was used for all assessments with the exception of the screening biopsy, which used paraffin block, if available. An independent central laboratory processed and stained the BM biopsies with hematoxylin and eosin, and for reticulin (Gordon and Sweet method) and collagen (Masson trichrome) to reduce preanalytical variation. Two hematopathologists (R.K.B. and A.O.) independently conducted the morphology review and assessed specimens for cellularity, megakaryocyte, erythroid, and myeloid quantity and appearance, trabecular bone quality, reticulin grade (MF-0 through MF-3) [17], and the presence of collagen. Differences in scoring were reconciled by rereview and discussion. 
Table 2. Patients with BM biopsies graded $\geq M F-2$ during the study

\begin{tabular}{|c|c|c|c|c|c|c|}
\hline 1 & Female; South/Central Asian & 48 & $\begin{array}{l}\text { Day -35: MF-1 } \\
\text { Day 310: MF-2 }\end{array}$ & $\begin{array}{l}\text { Negative } \\
\text { Negative }\end{array}$ & 75 & Yes; lack of efficacy \\
\hline 2 & Female; South/Central Asian & 25 & $\begin{array}{l}\text { Day -80: MF-0 } \\
\text { Day 399: MF-2 } \\
\text { Day 568: MF-0 }\end{array}$ & $\begin{array}{l}\text { Negative } \\
\text { Negative } \\
\text { Negative }\end{array}$ & 75 & Yes; increased ALT \\
\hline 4 & Female; East Asian & 18 & $\begin{array}{l}\text { Day -23: MF-0 } \\
\text { Day 365: MF-3 } \\
\text { Day 396: MF-0 } \\
\text { Day 732: MF-0 }\end{array}$ & $\begin{array}{l}\text { Negative } \\
\text { Positive } \\
\text { Negative } \\
\text { Negative }\end{array}$ & 50 & No \\
\hline
\end{tabular}

$\mathrm{BM}$, bone marrow; ALT, alanine aminotransferase; MF, marrow fibrosis.

a Biopsy performed 22 days posttreatment. ${ }^{\text {b }}$ Biopsy performed 288 days posttreatment.

\section{Results}

\section{Patient Disposition}

Of 186 patients screened, 167 were enrolled and received at least 1 dose of the study drug. Five patients from one center were excluded due to protocol/Good Clinical Practices violations. Of the 162 remaining patients, 118 (73\%) completed the study and $113(70 \%)$ completed $>22$ months of treatment with eltrombopag. Of the $48 / 162$ (30\%) patients who discontinued eltrombopag, 22 (14\%) discontinued due to adverse events (AEs), 11 (7\%) due to lack of efficacy, 7 (4\%) due to investigator discretion, and $8(5 \%)$ due to patient decision.

Of the AEs leading to discontinuation, only increased alanine aminotransferase (ALT) levels $(n=3)$, toxic hepatitis $(n=2)$, cerebral hemorrhage $(n=2)$, and increased blood bilirubin $(n=2)$ occurred in more than 1 patient each (Table 1). Information on the completion status was not available for $1(<1 \%)$ patient.

Three patients who discontinued eltrombopag had increased reticulin formation at the time of discontinuation (Table 2). A biopsy from patient 1 , who discontinued due to lack of efficacy, was graded MF-2 with no collagen. She had no AEs related to BM function, although there was no subsequent biopsy performed. Patient 2 , who discontinued on day 525 because of an ALT level that was more than 5 times the upper limit of normal, had a biopsy grad- ed MF-2; a biopsy on day 568 showed MF-1. Finally, a biopsy was graded MF-3 with the presence of collagen for patient 3, who discontinued treatment because she wanted to become pregnant. The follow-up biopsy 182 days later, on day 516, was graded MF-0 and negative for collagen. Biopsies from the remaining 2 patients with findings of MF-2/MF-3 and collagen who were not withdrawn from the study are also shown in Table 2.

\section{Demographics and Baseline Characteristics}

Demographic and baseline characteristics of the 162 patients are summarized in Table 3. The majority of patients $(63 \%)$ were between 18 and 49 years old, and the median age was 42 years (range 18-80). The overall study population comprised 50\% Caucasian and $49 \%$ Asian patients (20\% East Asian, 29\% Central/South Asian), and $1 \%$ had missing ethnicity data. A total of 155 patients (96\%) had an ITP diagnosis for $\geq 6$ months, and 124 (77\%) had an ITP diagnosis for $\geq 12$ months at baseline. A total of 114 patients $(70 \%)$ had received prior therapy for ITP, and 93 (57\%) were receiving ITP medication at baseline. Thirteen patients ( $8 \%$ ) had prior exposure to a TPO-RA, of whom 7 received eltrombopag and 4 received romiplostim $\geq 6$ months before enrollment. Approximately three quarters $(76 \%)$ of the patients had a baseline platelet count of $\leq 50 \times 10^{9} / \mathrm{L}$, and $23 \%$ of patients were splenectomized. 
Table 3. Patient demographics and baseline characteristics $(n=$ 162)

\begin{tabular}{ll}
\hline Median age, years (range) & $42.0(18-80)$ \\
Female, $n(\%)$ & $104(64)$ \\
Ethnicity, $n(\%)$ & \\
$\quad$ Caucasian & $81(50)$ \\
$\quad$ East Asian & $33(20)$ \\
$\quad$ Central/South Asian & $47(29)$ \\
Splenectomized, $n(\%)$ & $37(23)$ \\
$\geq 12$ months since ITP diagnosis, $n(\%)$ & $124(77)$ \\
Platelet counts, $n(\%)$ & \\
$\quad<15 \times 10^{9} / \mathrm{L}$ & $59(36)$ \\
15 to $<30 \times 10^{9} / \mathrm{L}$ & $34(21)$ \\
30 to $50 \times 10^{9} / \mathrm{L}$ & $31(19)$ \\
$>50 \times 10^{9} / \mathrm{L}$ & $38(23)$ \\
\hline
\end{tabular}

ITP, immune thrombocytopenia.

Table 4. Baseline and 2-year comparison of BM fibrosis grade in patients who all had 3 protocol-defined biopsies $(n=93)$

\begin{tabular}{llllll}
\hline $\begin{array}{l}\text { Baseline } \\
\text { grade }\end{array}$ & \multicolumn{2}{l}{ 2-year grade, $n(\%)$} & & & \\
\cline { 2 - 6 } & MF-0 & MF-1 & MF-2 & MF-3 & total \\
\hline MF-0 & $79(85)$ & $9(10)$ & 0 & 0 & $88(95)$ \\
MF-1 & $2(2)$ & $1(1)$ & 0 & 0 & $3(3)$ \\
MF-2 & 0 & 0 & 0 & 0 & 0 \\
MF-3 & 0 & 0 & 0 & 0 & 0 \\
Missing & $2(2)$ & 0 & 0 & 0 & $2(2)$ \\
\hline Total & $83(89)$ & $10(11)$ & 0 & 0 & $93(100)$ \\
\hline
\end{tabular}

$\mathrm{BM}$, bone marrow; MF, marrow fibrosis.

\section{Treatment}

The median daily dose of eltrombopag was $49.7 \mathrm{mg}$ (range 5-75 mg), and the median time on treatment was 104 weeks (range 2.4-113 weeks). The majority of patients $(61 \%)$ completed at least 24 months of eltrombopag treatment, and $73 \%$ completed more than 18 months of treatment.

\section{Analysis of BM Biopsies}

Baseline BM biopsies for 3 patients were not assessable, 2 because the sample was inadequate and 1 because of a procedural error. These patients were not included in the $\mathrm{BM}$ analysis. Of the 159 patients assessed at baseline, 150 (94\%) had MF-0 and 9 (6\%) had MF-1 (Fig. 1). No baseline biopsies were positive for collagen. Most patients' (96\%) on-treatment biopsies were normal or had

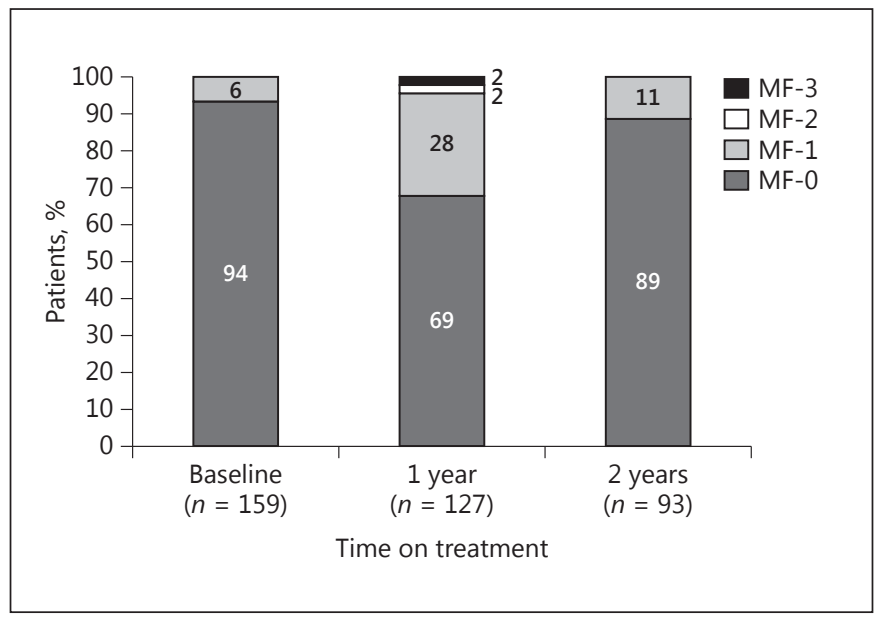

Fig. 1. Reticulin level at baseline and the 1- and 2-year assessments. MF grades at baseline and each on-treatment time interval were recorded using the European Consensus scale. On-treatment biopsies, performed within 14 days of the last dose of study medication, were categorized as 1 year if assessed between 10 and 22 months, and 2 years if assessed at $\geq 22$ months after treatment initiation.

minimally increased reticulin (graded as either MF-0 or MF-1, respectively).

Overall, 127 patients completed the baseline and 1-year BM biopsies, and 93 patients completed all $3 \mathrm{BM}$ biopsies (i.e., baseline and 1 and 2 years). At 1 year $(n=$ $127)$, biopsies of 87 patients (69\%) had a grade of MF-0, 35 (28\%) had MF-1, 3 (2\%) had MF-2, and 2 (2\%) had MF-3 (Fig. 1). At 2 years $(n=93), 83$ patients $(89 \%)$ had a grade of MF-0, 10 (11\%) had MF-1, and no patients had MF-2 or MF-3. Biopsies of 5 patients (4\%) at 1 year and 1 patient (1\%) at 2 years were positive for collagen.

Eight patients had either $\geq$ MF- 2 only $(n=2)$, the presence of collagen only $(n=3)$, or both $(n=3$; Table 2$)$. None of these patients had clinical symptoms typical of BM dysfunction or abnormalities of clinical concern reported in the white blood cell count or peripheral blood smear.

During the first year, 2 patients (2\%) had shifts from MF-0 to MF-2, and 2 patients (2\%) had shifts from MF-0 to MF-3. There was also 1 patient $(<1 \%)$ with a shift from MF-1 to MF-2. One patient was graded as MF-2 at 1 year and remained at MF- 2 at the posttreatment day 22 assessment. One patient was graded as MF-3 at 1 year, but an on-treatment biopsy 1 month later was graded as MF-0, as was a biopsy at 2 years. 
Table 5. Lineage-specific BM biopsy analysis at baseline and the 1- and 2-year assessments

\begin{tabular}{|c|c|c|c|c|c|c|c|c|c|}
\hline & \multicolumn{3}{|c|}{ Megakaryocytic } & \multicolumn{3}{|l|}{ Myeloid } & \multicolumn{3}{|l|}{ Erythroid } \\
\hline & $\mathrm{BL}$ & 1 year & 2 years & $\mathrm{BL}$ & 1 year & 2 years & $\mathrm{BL}$ & 1 year & 2 years \\
\hline Quantity, $n(\%)$ & 157 & 126 & 92 & 157 & 126 & 93 & 157 & 126 & 93 \\
\hline Normal & $12(8)$ & $2(2)$ & $2(2)$ & $140(89)$ & $98(78)$ & $75(81)$ & $140(89)$ & $112(89)$ & $82(88)$ \\
\hline Increased & $143(91)$ & $124(98)$ & $90(98)$ & $15(10)$ & $27(21)$ & $17(18)$ & $15(10)$ & $13(10)$ & $11(12)$ \\
\hline Decreased & $2(1)$ & 0 & 0 & $2(1)$ & $1(<1)$ & $1(1)$ & $2(1)$ & $1(<1)$ & 0 \\
\hline Quality, $n(\%)$ & 157 & 121 & 92 & 157 & 126 & 93 & 157 & 126 & 93 \\
\hline Normal maturation & $153(97)$ & $120(99)$ & $88(96)$ & $156(>99)$ & $124(98)$ & $87(94)$ & $156(>99)$ & $125(>99)$ & $93(100)$ \\
\hline Left shift & $4(3)$ & $1(<1)$ & $4(4)$ & $1(<1)$ & $2(2)$ & $6(6)$ & $1(<1)$ & $1(<1)$ & 0 \\
\hline
\end{tabular}

$\mathrm{BM}$, bone marrow; BL, baseline; $n$, number of patients with data available.

Table 6. Comparison of cellularity, trabecular bone quality, and collagen at baseline and the 1- and 2-year assessments

\begin{tabular}{lccc}
\hline & Baseline & 1 year & 2 years \\
\hline Cellularity, $n(\%)$ & 141 & 127 & 94 \\
Markedly hypocellular $(\leq 20 \%)$ & $1(<1)$ & $1(<1)$ & 0 \\
Moderately hypocellular $(30 \%)$ & $4(3)$ & $2(2)$ & $7(7)$ \\
Normal (40-60\%) & $127(90)$ & $101(80)$ & $71(76)$ \\
Moderately hypercellular (70\%) & $1(<1)$ & $11(9)$ & $10(11)$ \\
Markedly hypercellular $(\geq 80 \%)$ & $8(6)$ & $12(9)$ & $6(6)$ \\
\hline Trabecular bone quality, $n(\%)$ & 145 & 122 & 88 \\
Normal & $72(50)$ & $61(50)$ & $55(63)$ \\
Thinning & $73(50)$ & $61(50)$ & $33(38)$ \\
\hline Collagen, $n(\%)$ & 3 & 127 & 93 \\
Negative & $3(100)$ & $122(96)$ & $92(99)$ \\
Positive & 0 & $5(4)$ & $1(1)$ \\
\hline
\end{tabular}

$n$, number of patients with data available.

${ }^{1}$ Includes patients with any MF grade.

Compared with baseline, 79 out of 93 patients (85\%) had MF-0 at 2 years, $9(10 \%)$ had a 1 -grade increase, 2 (2\%) had a 1-grade decrease, 1 (1\%) remained MF-1, and none had 2- or 3-grade increases (Table 4). Data were missing for 2 (2\%) patients. None of the on-treatment biopsies was prompted by an abnormal peripheral blood smear or performed at the investigator's discretion for clinical symptoms suggestive of BM dysfunction.

In addition to grading for reticulin and collagen, BM specimens were assessed for cellularity, quantity, appearance of megakaryocytes, erythroid, and myeloid cells, and trabecular bone quality (Table 5). Megakaryocyte hyper- plasia was observed in the majority of patients (91\% at baseline and $98 \%$ at both years 1 and 2). Erythroid hyperplasia was observed in 10-12\% of patients, and myeloid hyperplasia was found in $10-21 \%$. Megakaryocyte, myeloid, and erythroid morphology was normal in most patients.

Cellularity was normal in 80,80 , and $76 \%$ of patients at baseline and the 1- and 2-year assessments, respectively (Table 6). Trabecular bone thinning was found in 50\% of patients at baseline, $50 \%$ at year 1 , and $38 \%$ at year 2 . No pathologic patterns of changes or clinically relevant changes in lineage features were identified in the ontreatment biopsies compared with baseline.

\section{Safety}

There were no BM fibrosis-related AEs reported. The most common AEs occurring in $>10 \%$ of patients were headache, cough, arthralgia, diarrhea, nausea, upper respiratory tract infection, and pyrexia (Table 7).

\section{Discussion}

This open-label safety study was designed to prospectively determine baseline levels of BM fibrosis in previously treated adults with persistent/chronic ITP and to evaluate the long-term effect of eltrombopag treatment on the formation of BM reticulin and/or collagen fibers. Six percent of patients had a BM fibrosis score of MF-1 at baseline. After 2 years of treatment, $86 \%$ of patients had no increase in grade of reticulin, and $10 \%$ had an increase of 1 grade in reticulin. No patient with an increase to MF-2 or the presence of collagen had clinical signs or symptoms indicative of BM dysfunction. 
Table 7. AEs occurring in $>10 \%$ of patients (safety population) $(n=162)$

\begin{tabular}{lr}
\hline AE & $n(\%)$ \\
\hline Any common event & $118(73)$ \\
Headache & $31(19)$ \\
Cough & $23(14)$ \\
Arthralgia & $22(14)$ \\
Diarrhea & $22(14)$ \\
Nausea & $21(13)$ \\
Upper respiratory tract infection & $21(13)$ \\
Pyrexia & $18(11)$ \\
Back pain & $17(10)$ \\
Fatigue & $17(10)$ \\
Gingival bleeding & $17(10)$ \\
Nasopharyngitis & $17(10)$ \\
Dyspepsia & $16(10)$ \\
Vomiting & $16(10)$ \\
\hline
\end{tabular}

AEs, adverse events.

$\mathrm{BM}$ biopsies are not routinely performed in the diagnosis or management of chronic ITP. Although some studies have retrospectively reviewed biopsy specimens, prospectively collected data in patients with chronic ITP are limited. A Danish study of 187 patients with chronic ITP never treated with TPO-RAs showed that $60 \%$ had MF- $0,39 \%$ had MF-1, and approximately $2 \%$ had MF-2 or MF-3 [18]. Whether the risk of BM fibrosis is due to the TPO-RAs themselves or the underlying disease remains unclear. Even in the absence of TPO-RAs, the results of several studies have revealed the presence of background BM reticulin fibrosis in patients with a diagnosis of ITP [6, 11, 18-20]. Furthermore, increased BM reticulin formation has been described in patients with refractory ITP, as well as in those with other autoimmune diseases [21,22].

TPO-RAs have been associated with increases in the formation of reticulin fibers $[11,13,19]$, but the lack of prospectively collected and consistently stained and analyzed pretreatment BM specimens has limited the robustness of the conclusions. An open-label study was conducted to assess the incidence of fibrosis after 3 years of treatment with romiplostim using the Bauermeister scale, and it also included a central review by 2 hematopathologists [23]. Patients were required to have normal $\mathrm{BM}$ at baseline and were assigned to 1 of 3 cohorts, with $\mathrm{BM}$ biopsies scheduled after 1,2, or 3 years of treatment rather than annual assessments in the same patients, as in the current study. After 2 and 3 years of treatment, 7 patients had increases in reticulin of $\geq 2$ grades, and specimens from 2 patients showed the presence of fibrosis. After discontinuing romiplostim, 3 out of 9 patients with BM changes had follow-up biopsies that showed normal BM.

In this prospective, longitudinal study, we found that most patients with chronic ITP had MF-0 at baseline, and most patients experienced no change or a mild increase in reticulin over 2 years of treatment with eltrombopag. Furthermore, our findings indicate that collagen fibrosis was reversible.

In conclusion, these data suggest that treatment with eltrombopag is generally not associated with clinically relevant increases in BM reticulin or collagen.

\section{Acknowledgements}

This work was supported by GlaxoSmithKline (GSK); eltrombopag is an asset of Novartis AG as of March 2, 2015. The authors wish to thank Christine K. Bailey, former employee of GSK at the time of the study, for her assistance with the data analysis for the manuscript and her critical review to previous drafts. Editorial support (assembling tables and figures, collating author comments, copyediting, fact checking, and referencing) and graphic services were provided by Prasad Kulkarni, PhD, William Perlman, PhD, and Nancy Price, PhD, of AOI Communications, L.P., and were funded by GSK and Novartis Pharmaceuticals Corporation.

\section{Author Contributions}

R.K.B. contributed to the conception and design of the study, acquisition of the data, and data analysis and interpretation; R.S.M.W. and A.O. contributed to the acquisition of the data and data analysis and interpretation; M.M.T. contributed to the acquisition of the data; K.K.B. contributed to the conception and design of the study and data analysis and interpretation; and P.B. and D.T. contributed to the data analysis and interpretation. All authors provided critical review of the manuscript and approval of the final version of the manuscript. All listed authors meet the criteria for authorship set forth by the International Committee for Medical Journal Editors.

\section{Disclosure Statement}

R.K.B. and A.O. received research funding from GlaxoSmithKline (GSK). R.S.M.W. received research funding from Bayer, Biogen-Idec, Bristol-Myers Squibb, GSK, Johnson \& Johnson, Merck Sharp \& Dohme, Novartis, Pfizer, and Roche; he serves as a consultant for Bayer, Biogen-Idec, and Novartis, serves on the speaker's bureau for Novartis, and has participated in advisory boards for Biogen-Idec and Novartis. K.K.B. and D.T. were employees of GSK during the time in which the study was conducted. P.B. was an employee of GSK during the study and initial publication development, and is currently an employee at Novartis. K.K.B., P.B., and D.T. hold equity ownership in GSK. 


\section{References}

1 Ballem PJ, Segal GM, Stratton JR, Gernsheimer T, Adamson JW, Slichter SJ: Mechanisms of thrombocytopenia in chronic autoimmune thrombocytopenic purpura. Evidence of both impaired platelet production and increased platelet clearance. J Clin Invest 1987;80:33-40.

2 McMillan R, Wang L, Tomer A, Nichol J, Pistillo J: Suppression of in vitro megakaryocyte production by antiplatelet autoantibodies from adult patients with chronic ITP. Blood 2004;103:1364-1369.

3 Bauermeister DE: Quantitation of bone marrow reticulin - a normal range. Am J Clin Pathol 1971;56:24-31.

4 Beckman EN, Brown AW Jr: Normal reticulin level in iliac bone marrow. Arch Pathol Lab Med 1990;114:1241-1243.

5 Mufti G, Bagg A, Hasserjian R, Bain B, Kuter D, Dreiling L, Nichol J: Bone marrow reticulin in patients with immune thrombocytopenic purpura. Blood 2006;108:3982.

6 Rizvi H, Butler T, Calaminici M, Doobaree IU, Nandigam RC, Bennett D, Provan D, Newland AC: United Kingdom immune thrombocytopenia registry: retrospective evaluation of bone marrow fibrosis in adult patients with primary immune thrombocytopenia and correlation with clinical findings. Br J Haematol 2015;169:590-594.

7 Bussel JB, Cheng G, Saleh MN, Psaila B, Kovaleva L, Meddeb B, Kloczko J, Hassani H, Mayer B, Stone NL, Arning M, Provan D, Jenkins JM: Eltrombopag for the treatment of chronic idiopathic thrombocytopenic purpura. N Engl J Med 2007;357:2237-2247.

8 Bussel JB, Kuter DJ, Pullarkat V, Lyons RM, Guo M, Nichol JL: Safety and efficacy of longterm treatment with romiplostim in thrombocytopenic patients with chronic ITP. Blood 2009; 113:2161-2171.

9 Cheng G, Saleh MN, Marcher C, Vasey S, Mayer B, Aivado M, Arning M, Stone NL, Bussel JB: Eltrombopag for management of chronic immune thrombocytopenia (RAISE): a 6-month, randomised, phase 3 study. Lancet 2011;377:393-402.
10 Saleh MN, Bussel JB, Cheng G, Meyer O, Bailey $\mathrm{CK}$, Arning M, Brainsky A; EXTEND Study Group: Safety and efficacy of eltrombopag for treatment of chronic immune thrombocytopenia: results of the long-term, open-label EXTEND study. Blood 2013;121: 537-545.

11 Brynes RK, Orazi A, Theodore D, Burgess P, Bailey CK, Thein MM, Bakshi KK: Evaluation of bone marrow reticulin in patients with chronic immune thrombocytopenia treated with eltrombopag: data from the EXTEND study. Am J Hematol 2015;90:598-601.

12 Ghanima W, Geyer JT, Lee CS, Orazi A, Boiocchi L, Imahiyerobo A, Bussel JB: Bone marrow fibrosis in immune thrombocytopenia (ITP) patients treated with thrombopoietin receptor agonists (TRA) - a single center long-term follow-up. Blood 2013;122:3527.

13 Ghanima W, Junker P, Hasselbalch HC, Boiocchi L, Geyer JT, Feng X, Gudbrandsdottir S, Orazi A, Bussel JB: Fibroproliferative activity in patients with immune thrombocytopenia (ITP) treated with thrombopoietic agents. $\mathrm{Br}$ J Haematol 2011;155:248-255.

14 British Committee for Standards in Haematology General Haematology Task Force: Guidelines for the investigation and management of idiopathic thrombocytopenic purpura in adults, children and in pregnancy. $\mathrm{Br} \mathrm{J}$ Haematol 2003;120:574-596.

15 Neunert C, Lim W, Crowther M, Cohen A, Solberg L Jr, Crowther MA; American Society of Hematology: The American Society of Hematology 2011 evidence-based practice guideline for immune thrombocytopenia. Blood 2011;117:4190-4207.

16 Provan D, Stasi R, Newland AC, Blanchette VS, Bolton-Maggs P, Bussel JB, Chong BH, Cines DB, Gernsheimer TB, Godeau B, Grainger J, Greer I, Hunt BJ, Imbach PA, Lyons G, McMillan R, Rodeghiero F, Sanz MA, Tarantino M, Watson S, Young J, Kuter DJ: International consensus report on the investigation and management of primary immune thrombocytopenia. Blood 2010;115: $168-186$.
17 Thiele J, Kvasnicka HM, Facchetti F, Franco V, van der Walt J, Orazi A: European consensus on grading bone marrow fibrosis and assessment of cellularity. Haematologica 2005; 90:1128-1132.

18 Ettrup MS, Jensen AØ, Engebjerg MC, Farkas DK, Nørgaard M, Cha S, Zhao S, Johansen P, Sørensen HT: Bone marrow reticulin and collagen content in patients with adult chronic immune thrombocytopenic purpura: a Danish nationwide study. Am J Hematol 2010;85: 930-934.

19 Ghanima W, Geyer JT, Lee CS, Boiocchi L, Imahiyerobo AA, Orazi A, Bussel JB: Bone marrow fibrosis in 66 patients with immune thrombocytopenia treated with thrombopoietin-receptor agonists: a single-center, longterm follow-up. Haematologica 2014;99:937944.

20 Kuter DJ, Mufti GJ, Bain BJ, Hasserjian RP, Davis W, Rutstein M: Evaluation of bone marrow reticulin formation in chronic immune thrombocytopenia patients treated with romiplostim. Blood 2009; 114:37483756.

21 Kim YK, Lee SS, Jeong SH, Ahn JS, Yang DH, Lee JJ, Kim HJ: Efficacy and safety of eltrombopag in adult refractory immune thrombocytopenia. Blood Res 2015;50:19-25.

22 Vergara-Lluri ME, Piatek CI, Pullarkat V, Siddiqi IN, O’Connell C, Feinstein DI, Brynes RK: Autoimmune myelofibrosis: an update on morphologic features in 29 cases and review of the literature. Hum Pathol 2014;45: 2183-2191.

23 Janssens A, Rodeghiero F, Anderson D, Chong BH, Boda Z, Pabinger I, Červinek L, Terrell DR, Wang X, Franklin J: Changes in bone marrow morphology in adults receiving romiplostim for the treatment of thrombocytopenia associated with primary immune thrombocytopenia. Ann Hematol 2016;95: 1077-1087. 